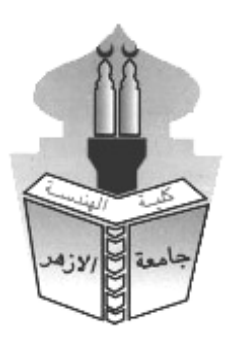

\title{
TWO PHASE MOTOR FED FROM SINGLE PHASE SUPPLY, USING CONTROL CIRCUIT
}

\author{
Sara Ali ${ }^{1}$, M. Adel ${ }^{2}$ and A. B. Kotb ${ }^{3}$ \\ ${ }^{1}$ Electrical Engineer \\ ${ }^{2}$ Faculty Of Engineering, Helwan University \\ ${ }^{3}$ Faculty Of Engineering, Al-Azhar University \\ Moabdelsamie45@yahoo.com, Khaledali-npp@yahoo.com

\begin{abstract}
The use of the two-phase stator windings with phase-angle control leaving the 2-phase voltages equal in magnitudes, which leads to better utilization of the machine material. The operation of 2-phase motor from single phase supply carried out by connecting one stator phase directly to the voltages source, with exciting the second stator phase through the phase shifting element. Minimum calculation effort for the two-phase stator currents and the motor electromagnetic torque acting on the rotor are determined. This calculation is carried out, using the time phase angle as parameter. In this method, the starting and the maximum torque values can be controlled by changing the stator field
\end{abstract} \\ from a pulsating of $\left(0^{\circ}\right)$ to a pure rotating field at $\left(90^{\circ}\right)$. \\ Keywords : Frequency, Motor Speed, Torque,Time Phase Angel, Motor \\ Sync.Spee

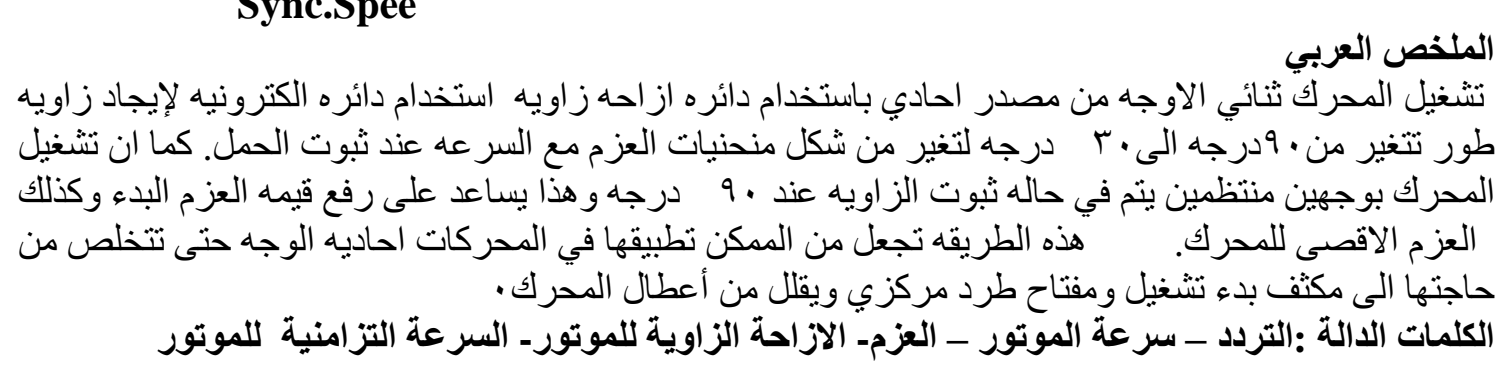

\section{INTRODUCTION}

Single-phase motors with main and auxiliary stator windings are widely used, but their extension into symmetrical two-phase enables higher rating range for the same motor. This also, allows good starting conditions with higher maximum torque. In addition to the conventional two-phase applications, the phase angle control $(2,3)$ will lead to more development of this modified motor. The gradual change of this motor speed can be achieved by controlling the time phase angle between the voltages of the stator windings. Keeping the two-phase voltages with equal in magnitudes enables better utilization of the machine materials.

The operation of 2-phase motor from single phase supply carried out by connecting one stator phase continuously to the voltages source, and exciting the second phase through a phase shifting circuit. In order to completely describe the motor performance for a given phase angle, it is necessary to derive an expression for the stator currents and the motor torque. By using the method of symmetrical components, the unbalanced stator phase voltages are resolved into two balanced components, positive and negative sequence values. Consequently, the forward and backward current components, which enable the motor performance can be determined.

The characteristics are calculated and plotted for different values of the phase angle between the two phase voltages. 


\section{OPERATION OF TWO-PHASE MOTOR FROM SINGLE PHASE SUPPLY}

The conventional capacitor-start, single-phase induction motor has two windings, the main winding and the auxiliary winding. A capacitor is placed in series with the auxiliary winding, thus nearly, a 90o difference phase angle between the two currents may be obtained. Consequently, a starting torque is developed and the motor becomes a self-starting motor. After the motor starts, the auxiliary winding is disconnected usually by means of centrifugal switch that operates at about 75 percent of synchronous speed. Finally the motor runs depending only on the main winding. Since this is being singlephase, some level of humming noise is associated with motor during the running.

To run this single-phase induction motor from two-phase supply, modification must be done. Capacitor and centrifugal switch are then not needed, and they are cut off through the operation. The auxiliary winding of the single phase motor is made of thin wire compared to that of its main winding which usually has copper wire of size corresponding to the load current. At normal operating condition, the auxiliary winding is connected to the voltage supply only for a few seconds, during the motor starting.

So, transformation is made in the auxiliary windings to have the same diameter for its copper wire with the same number of the phase turns as that of the main phase coil.

In the modified two phase motor, the windings will completely have a new design for the two-phase motor operation. Fig. (1), gives the modification of single-phase motor to be two-phase induction motor. Normally, a variable two phase voltage, variable frequency supply can be obtained from a single phase-two phase inverter to feed the modified motor with controlling its speed.

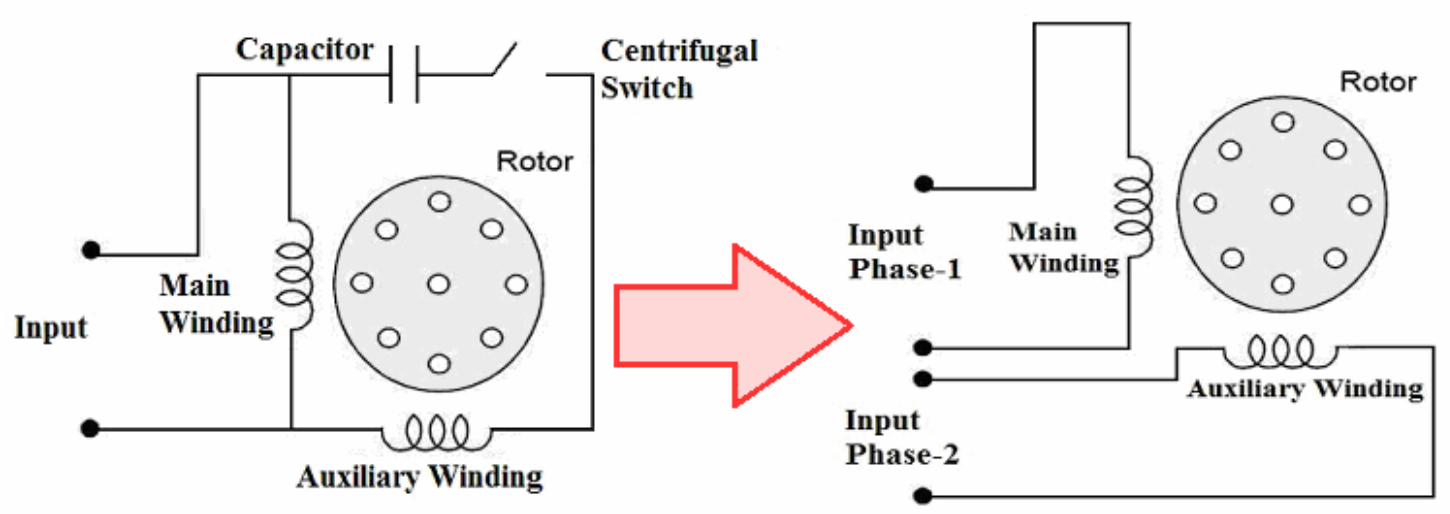

Fig. (1), Conversion of single phase to be two phase motor

\section{MOTOR PERFORMANCE UNDER CONTROLLED SUPPLY}

In order to completely describe the motor performance under general operating conditions of unbalanced supply voltage, it is necessary to use equivalent circuit obtained for forward and backward system. The resulting current Is 1 and Is2 in the first and second stator phase windings produce magneto motive forces, which are proportional to Is1Ws1 and Is $2 \mathrm{Ws} 2$, respectively. By using the shown equivalent circuit of Fig. (2), and the method of symmetrical components, the motor performance can be obtained. The unbalanced stator phase voltages are resolved into two balanced components, the positive and negative sequence values. Consequently, the forward and backward current components, which enable the motor performance are determined. 


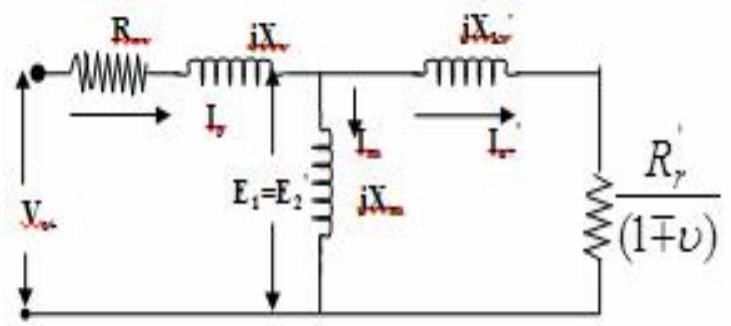

Fig (2) Equivalent circuit of unsymmetrical two phase induction motor.

\section{MATHEMATICAL ANALYSIS AND RESULTS}

Since, the method of time phase control is used, the second phase voltage $V_{2}$ is related to the first phase voltage $V_{1}$ with a time phase angle $\beta$ as:

$$
\mathrm{V}_{2}=\mathrm{V}_{1} \mathrm{e}^{\mathrm{j} \beta}
$$

Starting from the obtained equivalent circuit of Fig. (2), it enables now the calculations of the motor currents, the torque and the efficiency, for the values of the phase angle $\beta$. The expressions of positive and negative sequence current components can be written as: $\quad \mathrm{I}_{\mathrm{sf}}=\left(\mathrm{V}_{1}+\mathrm{j} \mathrm{V}_{2}\right) / 2 \mathrm{Z}_{\mathrm{f}}, \quad \mathrm{I}_{\mathrm{sb}}=\left(\mathrm{V} 1-\mathrm{j} \mathrm{V}_{2}\right) / 2 \mathrm{Z}_{\mathrm{b}}$

Where $\mathrm{Z}_{\mathrm{f}}$ and $\mathrm{Z}_{\mathrm{b}}$ are the positive and the negative sequence impedances given from Fig. (2). Therefore, the stator phase currents can be obtained. $\mathrm{I}_{\mathrm{s} 1}=\left(\mathrm{V}_{1} / 2\right)\left[(1+\sin \beta+\mathrm{j} \cos \beta) / \mathrm{Z}_{\mathrm{f}}+(1-\sin \beta-\mathrm{j} \cos \beta) / \mathrm{Z}_{\mathrm{b}}\right]$ $\mathrm{I}_{\mathrm{s} 2}=\left(\mathrm{V}_{1} / 2\right)\left[(1+\sin \beta+\mathrm{j} \cos \beta) / \mathrm{Z}_{\mathrm{f}}-(1-\sin \beta-\mathrm{j} \cos \beta) / \mathrm{Z}_{\mathrm{b}}\right]$

The calculations were carried out on a motor having the following parameters: $\mathrm{V}_{1}=220 \mathrm{v}, \mathrm{f}=50 \mathrm{~Hz}, \mathrm{R}_{\mathrm{s}}=3 \Omega, \mathrm{X}_{\mathrm{s} 1}=5.5 \Omega$ and $\mathrm{X}_{\mathrm{m}}=75 \Omega$.

The two phase stator windings are identical, and the stator phase currents are calculated. The results of the control current are plotted as function of the relative speed $v=n / n_{s}$, or the motor slip $\mathrm{s}$ with different value of dimensionless signal coefficient $\mathrm{a}_{1}=\sin (\beta)$, in Fig. (3), with using the phase angle $\beta$ as parameter.

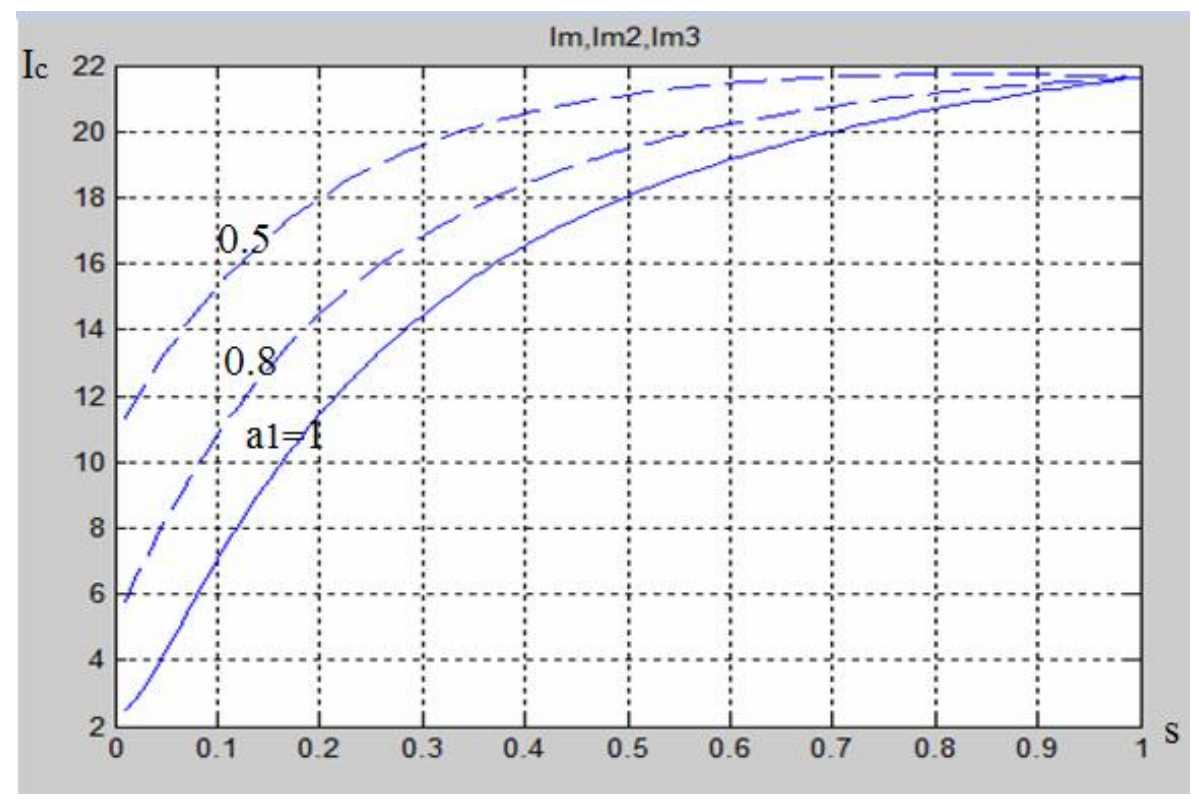

Fig. (3) The stator phase current as function of slip.

It is clear from the results, that the stator phase current Ic, has different values, while its maximum value obtained at only $\beta=\pi / 2$ at rotating field. 
It is evident that the small losses is obtained here and the corresponding high efficiency occurs when the field is a pure rotating.

The stator phase currents are known, then the electric loading may be determined and the motor performance characteristics can be obtained. The air gap power transferred from the stator to the rotor can be given

$\mathrm{Pg}=2\left[\mathbf{I}_{\mathrm{rf}}\left(\mathrm{R}_{\mathrm{r}} / \mathrm{s}\right)-\mathbf{I}^{2}{ }_{\mathrm{rb}} \mathrm{R}_{\mathrm{r}} /(2-\mathrm{s})\right]$

With $\mathrm{I}_{\mathrm{r}}, \mathrm{I}_{\mathrm{rb}}$ are the forward and backward components of the rotor currents. The electromagnetic torque may be then expressed as

$\mathrm{T}=\mathrm{P}_{\mathrm{g}} /(2 \pi \mathrm{f} / \mathrm{p})$

The developed torque is calculated and plotted as function of the relative speed $v$ or the slip s in Fig. (4), with different value of coefficient $a_{1}=\sin (\beta)$, for the phase angle $\beta$ is taken as parameter.

It is evident that the phase angle control gives a wide control range for the motor developed torque where $\beta$ changes from $90^{\circ}$ to $0.0^{\circ}$.

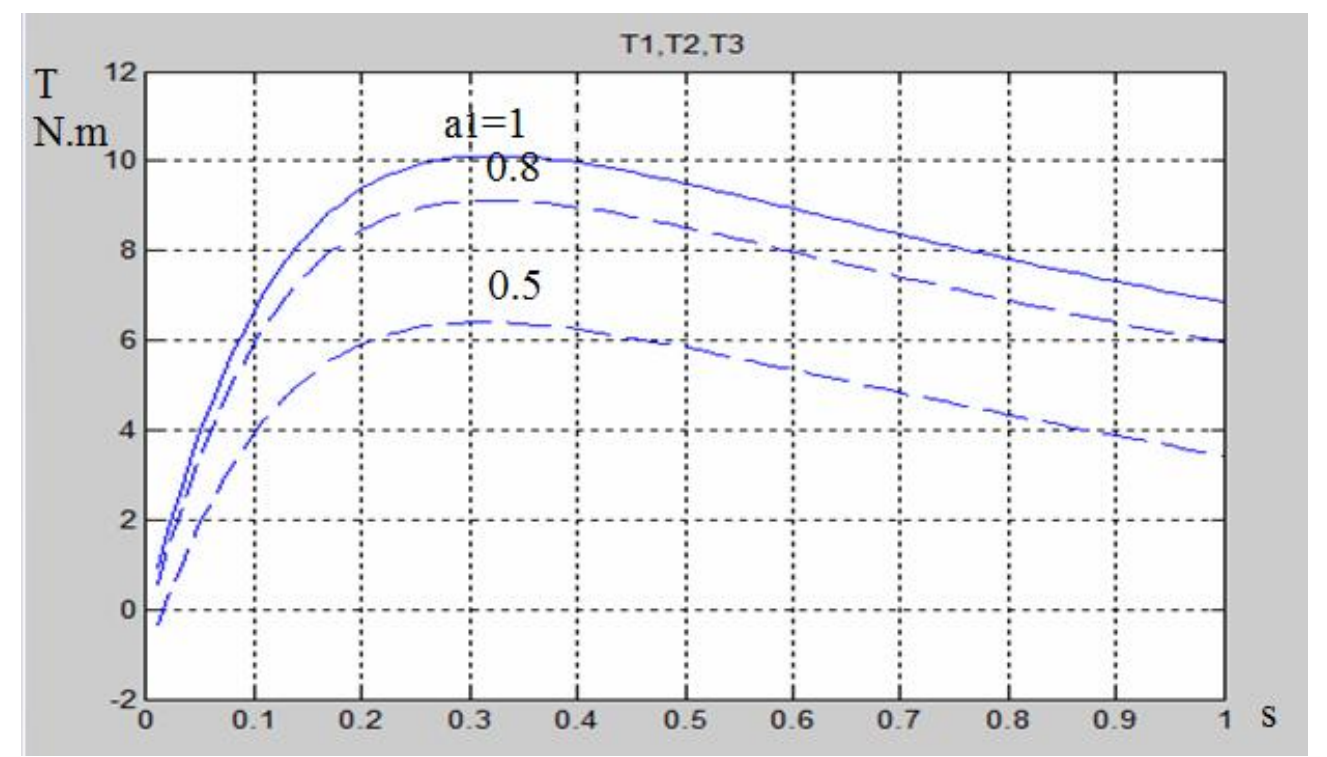

Fig. (4) The torque-slip characteristics with $\beta$ as parameter

The intersections of the constant load torque lines with the developed torque curves, can be determine the motor speed as function of the phase angle $\beta$. Fig. (4). Shows the motor developed torque as a function of the slip. Higher starting torque ranges are obtained by changing the field from pulsating into an almost rotating field, by increasing the phase angle.

It is noted that, the reduction in the phase angle control is carried out to obtain a gradual changes in the speed. This is associated with some reduction in the pullout torque which limits the value of rated load torque.

\section{CONCLUSIONS}

The better utilization of the motor materials, during all operating conditions can be achieved. It is carried out by using phase angle between the two phase stator voltages. The speed and the starting torque in a two-phase I.M can be changed over a wide range by changing the stator field from rotating at $\beta=\pi / 2$ to a pulsating field at $\beta=0.0$. The higher starting torque, efficiency, and speed are realized near the rotating field operation. 
The results of the simplified analysis carried out here, give the controlled current and torque expressions. In order to insure a suitable speed control and a large over load capacity the phase angle must be $\pi / 2$.

\section{REFERENCES}

1. Kotb, A. B. (1989). The Effects Of Secondary Sheet Design On The Performances Of Sleeve Rotor Induction Motor. AEC, Vol. 9(1), pp. 47-55.

2. SHALABY, M. (1985). Phase-Angle Control Of The Repulsion/Drive Secondary. IEEE Proceeding, Vol. 132, pp. 81-86.

3. SALEH, M. A., SHEHATA, M. A. (1973). Effect Of Method Of Control On The Transfer Function Parameters Of A Two-Phase Servo Motor. Measurement And Control, Vol. 6, pp. 517-524.

4. El-Sayad, A. M. (1989). Derivation Of Equivalent Account. Aec.1, Vol. 9, pp. 7393.

5. Ayman M. A., Salama, Zaid, A., Akram, H. A. (2018). Modified Single Phase Induction Motor Fed From The Solar Energy. Journal Of Engineering And Applied Sciences,Vol. 13(16), pp. 6904-6909.

6. Godbole, P., Fadnis A. Y. (2017). Inverters For Two Phase Induction Motor. Proceeding Of The 2017 International Conference On Power And Embedded Drive Control (Icpedc), pp. 484-489.

7. Farouk, A. E. (1990). An Analytical Solution For The Field Of A Hysteresis Motor Based On Complex Permeability. Ieee Transactions On Energy Conversion, Vol. 5(1), pp. 156-163.

8. Kotb, A. B, Shalaby, M. (1987). Analysis Of The Disk Rotor I.M. Amse Confer, Vol. 2, pp. 73-86.

9. Rungwicha, C, Vijit, K., Supat, K. (2017). Comparison Of Vector Control Of TwoPhase Induction Motor Using Continuous And Discontinuous Svpwm. International Electrical Engineering Congress, pp. 1-4. 